\title{
A New and Efficient Method for the Synthesis of Pyrimido[2,1-b]benzothiazole Derivatives
}

\author{
Fatemeh Chadegani, Fatemeh Darviche, Saeed Balalaie* \\ Peptide Chemistry Research Center, K. N. Toosi University of Technology, Tehran, Iran \\ Email: "balalaie@kntu.ac.ir
}

Received January 17, 2012; revised February 20, 2012; accepted March 15, 2012

\begin{abstract}
The one-pot three-component reaction of 2-aminobenzothiazole, benzaldehyde derivatives and $\beta$-ketoester, $\beta$-diketone or malonate derivatives in solvent-free conditions provides the corresponding pyrimido[2,1- $b]$ benzothiazole derivatives at $60^{\circ} \mathrm{C}$ in $60 \%-72 \%$ yields without using any catalyst in an optimistic time.
\end{abstract}

Keywords: 4H-Pyrimido[2,1-b]benzothiazole; Tandem Knoevenagel-Michael Reaction; One-Pot Reaction; Green Chemistry

\section{Introduction}

Fused heterocyclic compounds are very important compounds partially because of their pharmacological properties which include wide applications in medicinal chemistry [1]. Nowadays, much attention has been drawn to pyrimidines and condensed pyrimidine compounds for their worthwhile and interesting biological properties [2].

Pyrimido[2,1-b]benzothiazole derivatives are evaluated for their High affinity central benzodiazepine recep tor ligands $[3,4]$. The pharmaceutical properties of these ligands range from anxiolytic/anticonvulsant for agonists to antigenic/convulsant for inverse agonists and it has been used for treating patients diagnosed with epilepsy. Additionally, these compounds are incorporated with pyrazole structure known to possess tranquilizing, psychoanalytic and muscle relaxant activities [5-7]. Pyrimidobenzothiazole derivatives have also been known for their antimicrobial properties [8-10], anti-allergy [11], antitumor and anti-viral activities [12]. Meanwhile, oxopyrimido benzothiazoles have been assessed for bronchodilators, and bronchial asthma treatment [13]. Besides, these types of compounds, especially those with amide groups, show incredibly potent anti-inflammatory, anticoagulant, anti-fungicidal and anti-herbicidal activities and are used in the chemotherapy of carcinoid patients [14-17].

Therefore, a variety of effective strategies have been developed for the synthesis of these compounds. Most of the methods depict synthesis of pyrimido[2,1- $b]$ benzothiazole derivatives from 2 -amino benzothiazoles and $\beta$-haloesters [8-10], orthoesters [14-17], allenic [18] and ${ }^{*}$ Corresponding author. acetylenic groups [19-25]. Even though in some methods $\beta$-ketosters [26,27], $\alpha$-haloacids [28] and malonates [29] have been used.

Although the above methods that have been described for the synthesis of pyrimido[2,1-b]benzothiazole derivatives have their own advantages, but many of these reported procedures lead to some disadvantages including low yields, prolonged reaction time, various use of reagents, catalysts, toxic organic solvents in the reaction media as well as high temperature during completion of the reaction. Recently, solvent free conditions has received considerable interest ascribed to increasing of Global concerns over harmful chemical reagents and replacement of noxious organic solvents is one of the most important goals in green chemistry.

In continuation of our research programs to find novel one-pot multicomponent reactions [30-35] herein, we wish to report the one-pot three component reaction of benzaldehyde derivatives, active methylene compounds and 2-amino benzothiazole in solvent-free conditions. (Scheme 1).

\section{Results and Discussion}

In an easy and expedient procedure, along with proper conditions, Benzaldehyde derivatives 1, $\beta$-ketosters 2, $\beta$ diketones $\mathbf{3}$, malonates $\mathbf{4}$ and 2 -aminobenzothiazole $\mathbf{5}$ are used to synthesize $4 H$-pyrimido [2,1-b] benzothiazole and 2-oxo-pyrimido[2,1-b]benzothiazole compounds without using any solvent or catalyst. The corresponding products 6a-g has been achieved after 3 - $5 \mathrm{hr}$ with good yields (Table 1).

We have introduced a one-pot three-component con- 

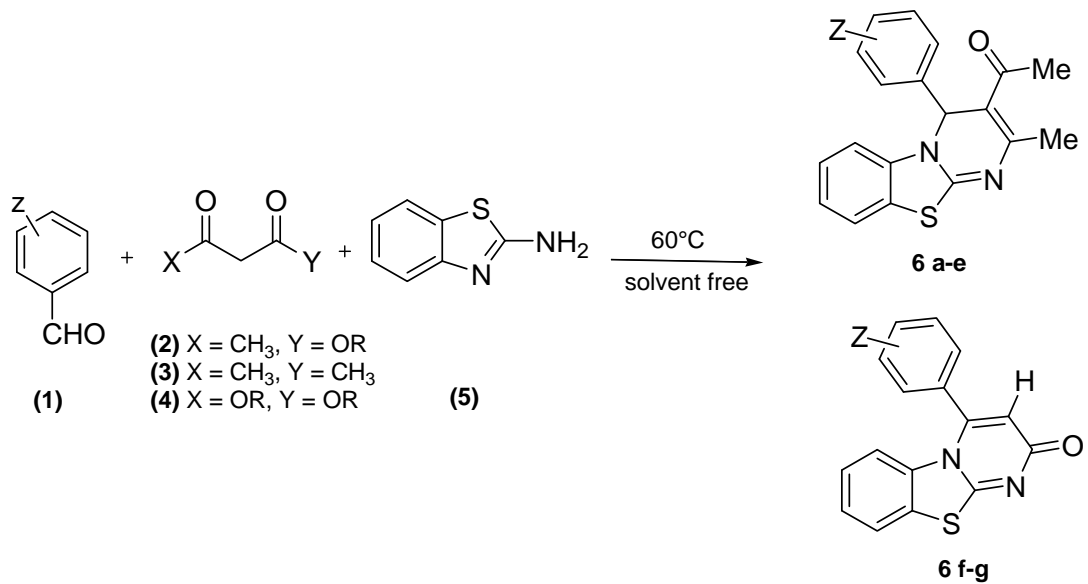

Scheme 1. Synthesis of pyrimido[2,1-b]benzothiazole derivatives 6a-g.

Table 1. One-pot synthesis of pyrimido[2,1-b]benzothiazolesin the $60^{\circ} \mathrm{C}$ in the solvent-free conditions .

\begin{tabular}{cccccc}
\hline Entry & $\mathbf{X}$ & $\mathbf{Y}$ & $\mathbf{Z}$ & Product & Yield (\%) $^{\dagger}$ \\
\hline $\mathrm{A}$ & $\mathrm{Me}$ & $\mathrm{OMe}$ & $3-\mathrm{OH}$ & $\mathbf{6 a}$ & 62 \\
$\mathrm{~B}$ & $\mathrm{Et}$ & $\mathrm{OEt}$ & $3-\mathrm{OH}$ & $\mathbf{6 b}$ & 64 \\
$\mathrm{C}$ & $\mathrm{Et}$ & $\mathrm{OEt}$ & $3-\mathrm{NO}_{2}$ & $\mathbf{6 c}$ & 60 \\
$\mathrm{D}$ & $\mathrm{Et}$ & $\mathrm{OEt}$ & $4-\mathrm{OH}$ & $\mathbf{6 d}$ & 63 \\
$\mathrm{E}$ & $\mathrm{Me}$ & $\mathrm{Me}$ & $3-\mathrm{OH}$ & $\mathbf{6 e}$ & 60 \\
$\mathrm{~F}$ & $\mathrm{OEt}$ & $\mathrm{OEt}$ & $4-\mathrm{OH}$ & $\mathbf{6 f}$ & 69 \\
$\mathrm{G}$ & $\mathrm{OMe}$ & $\mathrm{OMe}$ & $4-\mathrm{OH}$ & $\mathbf{6 f}$ & 69 \\
$\mathrm{H}$ & $\mathrm{OEt}$ & $\mathrm{OEt}$ & $2-\mathrm{OH}-5-\mathrm{Br}$ & $\mathbf{6 g}$ & 72 \\
\hline
\end{tabular}

${ }^{*}$ Reaction time in all reactions was between $3-5 \mathrm{hr} ;{ }^{\dagger}$ Isolated Yields.

densation reaction (MCR) that is one of the most useful methods for the synthesis of organic compounds in an optimistic time and only in a single step. Moreover, the aforementioned reaction most often leads to product that can be easily separated and purified by simple filtering and washing out with a regular solvent. In fact, we have not established a mechanism for the formation of these two compounds although a proposed mechanism is indicated in Schemes 2 and 3.

It seems benzaldehyde as an electrophile and $\beta$-ketosters (2) $/ \beta$-diketones (3), malonate (4) derivatives as active methylene compounds take part through an in-situ Knoevenagel reaction and an alkene is primarily formed. Afterwards, during the Michael addition reaction, 2aminbenzothiazole as a Michael donor attacks alkene during nucleophilic reaction, so an iminium ion is formed, subsequently with a proton transformation and an intramolecular cyclization, products $\mathbf{6 a - g}$ are produced. In the final step, during intramolecular cyclization, malonates with two proper leaving groups (two alkoxy groups), with considering the temperature, easily omit carbon dioxide from their structure and give rise to the formation of compounds 6f-h (2-oxo-pyrimido[2,1-b]benzotiazole de- rivatives). Schemes 1 and 2 show the proposed mechanisms for the formation of products.

The structures of compounds 6a-g were deduced from their ${ }^{1} \mathrm{H}$ NMR, ${ }^{13} \mathrm{C}$ NMR and IR spectral data and also by mass spectrometry. The 6a-e products exhibited a singlet in ${ }^{1} \mathrm{H}$ spectra at about $\delta=5.54-6.33 \mathrm{ppm}$ for H-4 and also a distinguished peak at $\delta=50.9-56.5 \mathrm{ppm}$ for C-4 in ${ }^{13} \mathrm{CNMR}$ spectroscopy. The $\mathbf{6 f - g}$ products demonstrated a singlet in ${ }^{1} \mathrm{H}$ spectra at $\delta=9.02-10.2 \mathrm{ppm}$ for $\mathrm{H}-3$ and also a distinguished peak at $\delta=110.9-116.2$ ppm for C-3 in ${ }^{13} \mathrm{CNMR}$ spectroscopy. The mass spectra of these compounds displayed molecular ion peaks. The selected spectroscopic data are reported in the experimental section.

\section{Conclusion}

As a result, $4 H$-pyrimido[2,1-b]benzothiazole (6a-e) and 2-oxo-pyrimido[2,1-b]benzothiazole (6f-g) are formed smoothly with the reaction of $\beta$-ketosters, $\beta$-diketone, malonates and benzaldehyde derivatives in the solventfree conditions with no solvent as well as no catalyst and subsequent annulation's reactions proceeded in accept- 


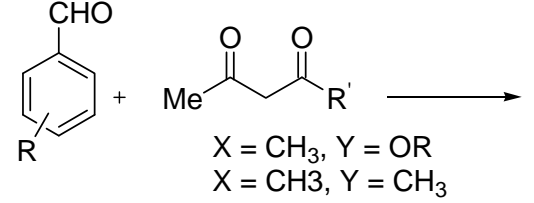

(1)<smiles>[R]c1ccc(C=C(C(C)=O)C(=O)CCCCCCC)cc1</smiles>

(7)
(5)<smiles>[R][R]1ccc(C(C(C([R])=O)=C(C)[O-])n2c(=[NH2+])sc3ccccc32)cc1</smiles>

(8)

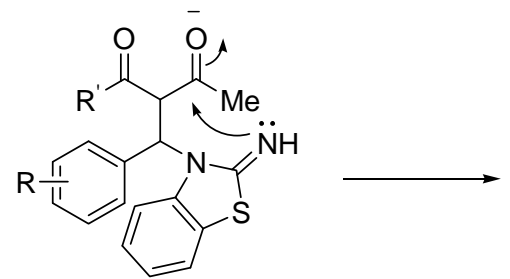

(9)<smiles></smiles>

6 (a-e)

Scheme 2. Proposed mechanism for the synthesis of $\mathbf{4 H}$-pyrimido[2,1-b]benzothiazole derivatives 6a-e in the solvent-free conditions.

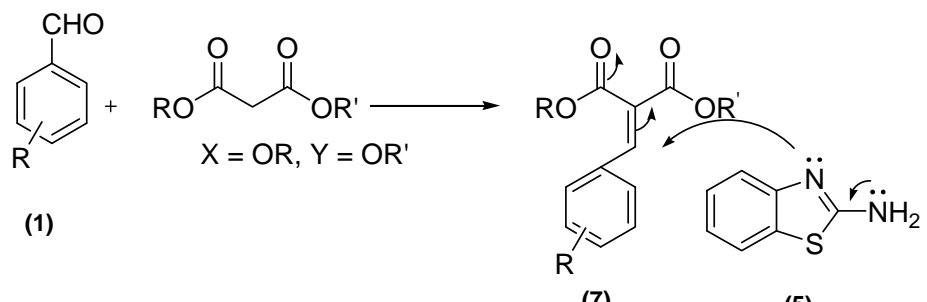

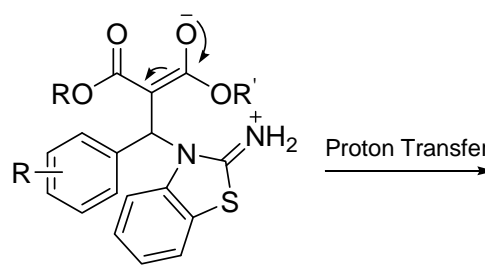

(8)<smiles>[R]OC(=O)C1N[n+]2c(sc3ccccc32)NC1=O</smiles>

(10)

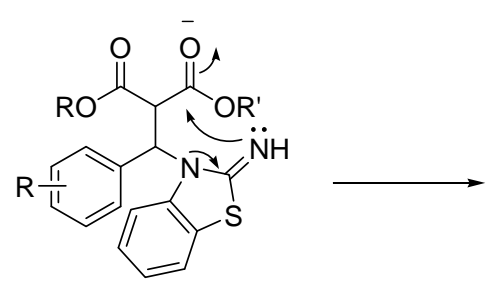

(9)<smiles>O=c1cc([Al])n2c(n1)sc1ccccc12</smiles>

6 (f-h)

Scheme 3. Proposed mechanism for the synthesis of 2-oxo-pyrimido[2,1-b]benzothiazole 6f-h derivatives in the solvent-free conditions. 
able yields. These derivatives present a class of compounds that can be used as procedures for the synthesis of new derivatives with useful biological activities. In addition, our method has significant advantages, such as the high bond forming efficiency, solvent-free reaction conditions.

\section{Experimental}

\subsection{Instruments and Characterization}

Melting points were recorded on an Electrothermal 9100 melting point apparatus and Infrared (IR) spectra were recorded on a ABB FTLA-2000 spectrophotometer using $\mathrm{KBr}$ disks. ${ }^{1} \mathrm{H}-\mathrm{NMR}$ and ${ }^{13} \mathrm{C}$-NMR spectra were recorded on Bruker DRX 500 (500 MHz) AVANCE spectrometer in DMSO using TMS as the internal standard. Mass spectra were recorded on HP 5379(Agilent Technology) (EI, 20eV, 70eV).

\subsection{General Procedure for the Synthesis of 4H-Pyrimido[2,1-b]benzothiazole-3-carboxylic Acid, 2-Methyl-4aryl-aklyl Ester:(6a-e)}

A mixture of 2-aminobenzothiazole (1 mmol, $152 \mathrm{mg}$ ) and benzaldehyde derivatives $(1 \mathrm{mmol})$ and ethylacetoacetate $(1 \mathrm{mmol}, 134 \mathrm{mg})$ or methylacetoactate $(1 \mathrm{mmol}$, $125 \mathrm{mg}$ ) or acetylacetone (1 mmol, $97 \mathrm{mg}$ ) were heated at $60^{\circ} \mathrm{C}$ in the solvent-free conditions for $4-5 \mathrm{hr}$. Completion of the reaction was confirmed by TLC (Petroleum ether:EtOAc 1:4). At the end of the reaction, the mixture was washed 3 times $(3 \times 20 \mathrm{ml})$ with water and diethylether. The desired products were obtained with high purity.

\subsection{General Procedure for the Synthesis of 4-Aryl-2H-pyrimido-[2,1-b]benzothiazol-2-one: (6f-g)}

A mixture of 2-aminobenzothiazole (1 mmol, $152 \mathrm{mg}$ ) and benzaldehyde derivatives $(1 \mathrm{mmol})$ and diethylmalonate $(1 \mathrm{mmol}, 160 \mathrm{mg})$ or dimethylmalonate $(1 \mathrm{mmol}$, $132 \mathrm{mg}$ ) was heated at $60^{\circ} \mathrm{C}$ in the solvent-free conditions for 3 - $3.5 \mathrm{hr}$. Completion of the reaction was confirmed by TLC (Petroleum ether: EtOAc 1:2). At the end of the reaction the mixture was washed 2 - 3 times with water and diethylether. The desired products were obtained with high purity. The purity of prepared compounds was tested by the elemental analysis of $\mathrm{C}, \mathrm{H}$, and $\mathrm{N}$ elements using a Heraus $\mathrm{CHN}$ rapid analyzer.

\subsection{Selected Data for Compounds 6a-g}

4H-Pyrimido[2,1-b]benzothiazole-3-carboxylic acid-2methyl-4(3-hydroxy phenyl)-methyl ester (6a, C19H16N2O2S):

$218 \mathrm{mg}$ (62\%), M.p.: $283^{\circ} \mathrm{C}-284^{\circ} \mathrm{C} ;{ }^{1} \mathrm{H}$ NMR (500 MHz,
DMSO- $\left.d_{6}\right): \delta=2.29\left(\mathrm{~s}, 3 \mathrm{H}, \mathrm{CH}_{3}\right), 3.61\left(\mathrm{~s}, 3 \mathrm{H}, \mathrm{CH}_{3}\right), 6.38$ $(\mathrm{s}, 1 \mathrm{H}, \mathrm{CH}), 6.58\left(\mathrm{~d}, 1 \mathrm{H}, J=10 \mathrm{~Hz}, \mathrm{H}_{\mathrm{Ar}}\right), 6.77(\mathrm{~s}, \mathrm{H}$, $\left.\mathrm{H}_{\mathrm{Ar}}\right), 6.82\left(\mathrm{~d}, 1 \mathrm{H}, J=10 \mathrm{~Hz}, \mathrm{H}_{\mathrm{Ar}}\right), 7.05(\mathrm{t}, 1 \mathrm{H}, J=13 \mathrm{~Hz}$, $\left.\mathrm{H}_{\mathrm{Ar}}\right), 7.18\left(\mathrm{t}, 1 \mathrm{H}, J=12 \mathrm{~Hz}, \mathrm{H}_{\mathrm{Ar}}\right), 7.28(\mathrm{t}, 1 \mathrm{H}, J=13 \mathrm{~Hz}$, $\left.\mathrm{H}_{\mathrm{Ar}}\right), 7.36\left(\mathrm{~d}, 1 \mathrm{H}, J=12.8 \mathrm{~Hz}, \mathrm{H}_{\mathrm{Ar}}\right), 7.73(\mathrm{~d}, 1 \mathrm{H}, J=12.8$ $\mathrm{Hz}, \mathrm{H}_{\mathrm{Ar}}$ ), 9.45 (s ,1H, OH)ppm; ${ }^{13} \mathrm{C}$ NMR $(125 \mathrm{MHz}$, DMSO- $\left.d_{6}\right): \delta=23.3,50.9,56.4,102.6,112.3,113.4$, $115.3,117.6,122.8,122.9,124.1,126.8,129.5,137.6$, $142.8,153.8,154.1,157.6,162.8,166.1 \mathrm{ppm}$; IR (KBr): $\bar{V}=2974,2608,1694,1607 \mathrm{~cm}^{-1}$; MS (EI, $\left.20 \mathrm{eV}\right)$ $\mathrm{C}_{19} \mathrm{H}_{16} \mathrm{~N}_{2} \mathrm{O}_{3} \mathrm{~S}, 352\left(78 \%, \mathrm{M}^{+\cdot}\right), 337\left(14 \%,[\mathrm{M}-\mathrm{Me}]^{+}\right)$, $321\left(8 \%,[\mathrm{M}-\mathrm{OMe}]^{+}\right), 293\left(51 \%,\left[\mathrm{M}-\mathrm{CO}_{2} \mathrm{Me}\right]^{+}\right), 259$ $\left(100 \%,\left[\mathrm{M}_{-} \mathrm{C}_{6} \mathrm{H}_{5} \mathrm{O}\right]^{+}\right), 199\left(45 \%,\left[\mathrm{M}-\mathrm{C}_{9} \mathrm{H}_{11} \mathrm{O}_{3}\right]^{+}\right)$; Anal. Calcd for $\mathrm{C}_{19} \mathrm{H}_{16} \mathrm{~N}_{2} \mathrm{O}_{3} \mathrm{~S}$ : C 64.76, H 4.58, N 7.95, Found C 64.60, H 4.43, N 7.85

4H-pyrimido[2,1-b]benzothiazole-3-carboxylic acid-2methyl-4(3-hydroxy phenyl)-ethyl ester (6b $\left.\mathbf{C}_{20} \mathbf{H}_{18} \mathbf{N}_{2} \mathbf{O}_{3} \mathbf{S}\right)$ :

$234 \mathrm{mg}(64 \%), \mathrm{Mp}=258^{\circ} \mathrm{C}-260^{\circ} \mathrm{C} ;{ }^{1} \mathrm{H}$ NMR $(500 \mathrm{MHz}$, DMSO- $\left.d_{6}\right): \delta=1.22\left(\mathrm{t}, J=7 \mathrm{~Hz}, 3 \mathrm{H}, \mathrm{CH}_{3}\right), 2.32(\mathrm{~s}, 3 \mathrm{H}$, $\left.\mathrm{CH}_{3}\right), 4.08\left(\mathrm{~m}, 2 \mathrm{H}, J=8 \mathrm{~Hz}, \mathrm{CH}_{2}\right), 6.39(\mathrm{~s}, 1 \mathrm{H}, \mathrm{CH})$, $6.60\left(\mathrm{~d}, 1 \mathrm{H}, J=8 \mathrm{~Hz}, \mathrm{H}_{\mathrm{Ar}}\right), 6.80\left(\mathrm{~s}, 1 \mathrm{H}, \mathrm{H}_{\mathrm{Ar}}\right), 6.87(\mathrm{~d}, 1 \mathrm{H}$, $\left.J=8 \mathrm{~Hz}, \mathrm{H}_{\mathrm{Ar}}\right), 7.07\left(\mathrm{t}, 1 \mathrm{H}, J=8 \mathrm{~Hz}, \mathrm{H}_{\mathrm{Ar}}\right), 7.2(\mathrm{t}, 1 \mathrm{H}, J=$ $\left.7.5 \mathrm{~Hz}, \mathrm{H}_{\mathrm{Ar}}\right), 7.32\left(\mathrm{t}, 1 \mathrm{H}, J=7.5 \mathrm{~Hz}, \mathrm{H}_{\mathrm{Ar}}\right), 7.38(\mathrm{~d}, 1 \mathrm{H}, J$ $\left.=8 \mathrm{~Hz}, \mathrm{H}_{\mathrm{Ar}}\right), 7.76\left(\mathrm{~d}, 1 \mathrm{H}, J=8 \mathrm{~Hz}, \mathrm{H}_{\mathrm{Ar}}\right), 9.45(\mathrm{~s}, 1 \mathrm{H}$, OH)ppm; ${ }^{13} \mathrm{C}$ NMR (125 MHz, DMSO- $\left.d_{6}\right): \delta=14.1,23.2$, $56.5,59.6,102.8,112,3,113.6,115.3,117.7,122.8$, $124.0,126.8,129.4,137.6,142.8,153.8,157.5,162.6$, 166.6 ppm;IR (KBr): $\bar{V}=2978,2587,1694,1597 \mathrm{~cm}^{-1}$; MS (EI, $20 \mathrm{eV}) \mathrm{C}_{20} \mathrm{H}_{18} \mathrm{~N}_{2} \mathrm{O}_{3} \mathrm{~S}, 366\left(60 \%, \mathrm{M}^{+\cdot}\right), 351-337$ $\left(25 \%,[\mathrm{M}-\mathrm{Et}]^{+\cdot}\right), 293\left(53 \%,\left[\mathrm{M}-\mathrm{CO}_{2} \mathrm{Et}\right]^{+\cdot}\right), 273(100 \%$, $\left[\mathrm{M}-\mathrm{C}_{6} \mathrm{H}_{5} \mathrm{O}\right]^{+\cdot}$ ), Anal. Calcd for $\mathrm{C}_{20} \mathrm{H}_{18} \mathrm{~N}_{2} \mathrm{O}_{3} \mathrm{~S}$ : C $65.55, \mathrm{H}$ 4.95, N 7.64, Found C 64.43, H 4.88, N 7.58.

4H-pyrimido[2,1-b]benzothiazole-3-carboxylic acid-2methyl-4(3-nitro phenyl)-ethyl ester (6c, $\mathrm{C}_{20} \mathbf{H}_{17} \mathbf{N}_{3} \mathbf{O}_{4} \mathrm{~S}$ ) $237 \mathrm{mg}(60 \%) ; \mathrm{Mp}=222^{\circ} \mathrm{C}-224^{\circ} \mathrm{C} ;{ }^{1} \mathrm{H}$ NMR $(500 \mathrm{MHz}$, DMSO- $\left.d_{6}\right): \delta=1.18\left(\mathrm{t}, 3 \mathrm{H}, J=8.3 \mathrm{~Hz}, \mathrm{CH}_{3}\right), 2.33(\mathrm{~s}, 3 \mathrm{H}$, $\left.\mathrm{CH}_{3}\right), 4.05\left(\mathrm{~m}, 2 \mathrm{H}, \mathrm{CH}_{2}\right), 6.69(\mathrm{~s}, 1 \mathrm{H}, \mathrm{CH}), 7.2(\mathrm{t}, 1 \mathrm{H}, J$ $\left.=11.5 \mathrm{~Hz}, \mathrm{H}_{\mathrm{Ar}}\right), 7.30\left(\mathrm{t}, 1 \mathrm{H}, J=11.5 \mathrm{~Hz}, \mathrm{H}_{\mathrm{Ar}}\right), 7.54(\mathrm{~d}$, $1 \mathrm{H}, J=14.5 \mathrm{~Hz}, \mathrm{H}_{\mathrm{Ar}}$ ), 7.59 (t, $1 \mathrm{H}, J=13.3 \mathrm{~Hz}, \mathrm{H}_{\mathrm{Ar}}$ ), $7.77\left(\mathrm{~d}, 1 \mathrm{H}, J=12 \mathrm{~Hz}, \mathrm{H}_{\mathrm{Ar}}\right), 7.85(\mathrm{~d}, 1 \mathrm{H}, J=13.5 \mathrm{~Hz}$, $\left.\mathrm{H}_{\mathrm{Ar}}\right) 8.08\left(\mathrm{~d}, 1 \mathrm{H}, J=13.5 \mathrm{~Hz}, \mathrm{H}_{\mathrm{Ar}}\right), 8.34\left(\mathrm{~s}, 1 \mathrm{H}, \mathrm{H}_{\mathrm{Ar}}\right)$; IR $(\mathrm{KBr}): \bar{V}=2962,1689,1607,1494 \mathrm{~cm}^{-1}$, Anal. Calcd for $\mathrm{C}_{20} \mathrm{H}_{17} \mathrm{~N}_{3} \mathrm{O}_{4} \mathrm{~S}$ : C 60.48, $\mathrm{H}$ 4.33, N 10.63, Found $\mathrm{C}$ 60.40, H 4.38, N 10.52 .

4H-pyrimido[2,1-b]benzothiazole-3-carboxylic acid-2methyl-4(4-hydroxy phenyl)-ethyl ester(6d,

$\left.\mathrm{C}_{20} \mathrm{H}_{18} \mathrm{~N}_{2} \mathrm{O}_{3} \mathrm{~S}\right)$

$230 \mathrm{mg}(63 \%) ; \mathrm{Mp}=267^{\circ} \mathrm{C}-269^{\circ} \mathrm{C} ;{ }^{1} \mathrm{H}$ NMR $(500 \mathrm{MHz}$, DMSO- $\left.d_{6}\right): \delta=1.18\left(\mathrm{t}, 3 \mathrm{H}, J=12 \mathrm{~Hz}, \mathrm{CH}_{3}\right), 2.3(\mathrm{~s}, 3 \mathrm{H}$, $\left.\mathrm{CH}_{3}\right), 4.03\left(\mathrm{~m}, 2 \mathrm{H}, \mathrm{CH}_{2}\right), 6.33(\mathrm{~s}, 1 \mathrm{H}, \mathrm{CH}), 6.62(\mathrm{~d}, 1 \mathrm{H}, J$ $\left.=14 \mathrm{~Hz}, \mathrm{H}_{\mathrm{Ar}}\right), 7.15\left(\mathrm{t}, 1 \mathrm{H}, J=12 \mathrm{~Hz}, \mathrm{H}_{\mathrm{Ar}}\right), 7.21(\mathrm{t}, 1 \mathrm{H}, J$ $\left.=14 \mathrm{~Hz}, \mathrm{H}_{\mathrm{Ar}}\right), 7.28\left(\mathrm{t}, 1 \mathrm{H}, J=12 \mathrm{~Hz}, \mathrm{H}_{\mathrm{Ar}}\right), 7.39(\mathrm{~d}, 1 \mathrm{H}, J$ $\left.=12.7 \mathrm{~Hz}, \mathrm{H}_{\mathrm{Ar}}\right), 7.71\left(\mathrm{~d}, 1 \mathrm{H}, J=12.7 \mathrm{~Hz}, \mathrm{H}_{\mathrm{Ar}}\right), 9.46(\mathrm{~s}$, $1 \mathrm{H}, \mathrm{OH}) \mathrm{pppm} ;{ }^{13} \mathrm{C} \mathrm{NMR}\left(125 \mathrm{MHz}, \mathrm{DMSO}-d_{6}\right): \delta=$ 
$14.1,23.2,56.2,59.4,103.1,112.4,115.1,122.8,122.8$, $123.9,126.7,128.4,132.2,137.7,153.6,157.3,162.4$, 165.65 ppm; IR (KBr): $\bar{V}=2$ 947, 1674, 1591, 1514 $\mathrm{cm}^{-1}$; MS (EI, $\left.20 \mathrm{eV}\right) \mathrm{C}_{20} \mathrm{H}_{18} \mathrm{~N}_{2} \mathrm{O}_{3} \mathrm{~S}, 366\left(47 \%, \mathrm{M}^{+\cdot}\right), 337$ $\left.\left.(22 \% \text {, [M-Et] }]^{+}\right), 293\left(100 \% \text {, [M-CO }{ }_{2} \mathrm{Et}\right]^{+}\right), 273(86 \%$, [M- $\left.\mathrm{C}_{6} \mathrm{H}_{5} \mathrm{O}\right]^{+}$). Anal. Calcd for $\mathrm{C}_{20} \mathrm{H}_{18} \mathrm{~N}_{2} \mathrm{O}_{3} \mathrm{~S}: \mathrm{C} 65.55, \mathrm{H}$ 4.95, N 7.64, Found C 64.45, H 4.90, N 7.60.

4H-pyrimido[2,1-b]benzothiazole-3-acetyl-2-methyl-4 (3-hydroxyphenyl) (6e, $\mathrm{C}_{19} \mathrm{H}_{16} \mathbf{N}_{\mathbf{2}} \mathrm{O}_{2} \mathrm{~S}$ )

$220 \mathrm{mg}(60 \%) ; \mathrm{Mp}=290^{\circ} \mathrm{C}-292^{\circ} \mathrm{C} ;{ }^{1} \mathrm{H}$ NMR $(500 \mathrm{MHz}$, DMSO-d $\left.d_{6}\right): \delta=2.29\left(\mathrm{~s}, 3 \mathrm{H}, \mathrm{CH}_{3}\right), 2.33\left(\mathrm{~s}, 3 \mathrm{H}, \mathrm{CH}_{3}\right)$, $6.51(\mathrm{~s}, 1 \mathrm{H}, \mathrm{CH}), 6.57\left(\mathrm{~d}, 1 \mathrm{H}, J=16 \mathrm{~Hz}, \mathrm{H}_{\mathrm{Ar}}\right), 6.79(\mathrm{t}$, $\left.1 \mathrm{H}, J=3.5 \mathrm{~Hz}, \mathrm{H}_{\mathrm{Ar}}\right), 6.85\left(\mathrm{~d}, 1 \mathrm{H}, J=13 \mathrm{~Hz}, \mathrm{H}_{\mathrm{Ar}}\right), 7.03(\mathrm{t}$, $\left.1 \mathrm{H}, J=13 \mathrm{~Hz}, \mathrm{H}_{\mathrm{Ar}}\right), 7.20\left(\mathrm{t}, 1 \mathrm{H}, J=13 \mathrm{~Hz}, \mathrm{H}_{\mathrm{Ar}}\right), 7.34(\mathrm{t}$, $\left.1 \mathrm{H}, J=13 \mathrm{~Hz}, \mathrm{H}_{\mathrm{Ar}}\right), 7.51\left(\mathrm{~d}, 1 \mathrm{H}, J=13 \mathrm{~Hz}, \mathrm{H}_{\mathrm{Ar}}\right), 7.75$ (d, $\left.1 \mathrm{H}, J=13 \mathrm{~Hz}, \mathrm{H}_{\mathrm{Ar}}\right), 9.43(\mathrm{~s}, 1 \mathrm{H}, \mathrm{OH}) \cdot \mathrm{ppm},{ }^{13} \mathrm{C} \mathrm{NMR}$ $\left(125 \mathrm{MHz}, \mathrm{DMSO}-d_{6}\right): \delta=13.1,56.5,59.2,103.3,112.3$, $113.2,115.7,117.2,121.8,123.7,126.8,129.4,136.3$, $142.3,153.7,157.5,161.4,166.8, \mathrm{IR}(\mathrm{KBr}): \bar{V}=2931$, 2608, 1617, 1590, $1499 \mathrm{~cm}^{-1}$; MS (EI, 20eV) $\mathrm{C}_{19} \mathrm{H}_{16} \mathrm{~N}_{2} \mathrm{O}_{2} \mathrm{~S}$, $336\left(65 \%, \mathrm{M}^{+}\right), 293\left(100 \%\right.$, [M-COMe] $\left.{ }^{+}\right), 243(100 \%$, [M- $\left.\mathrm{C}_{6} \mathrm{H}_{5} \mathrm{O}\right]^{+}$). Anal. Calcd for $\mathrm{C}_{19} \mathrm{H}_{16} \mathrm{~N}_{2} \mathrm{O}_{2} \mathrm{~S}: \mathrm{C} 67.83, \mathrm{H}$ 4.79, N 8.33, Found C 67.71, H 4.73, N 8.26.

4-(4-hydroxyphenyl)-2H-pyrimido-[2,1-b]benzothiazol2-one (6f, $\mathrm{C}_{16} \mathrm{H}_{10} \mathrm{~N}_{2} \mathrm{O}_{2} \mathrm{~S}$ )

$202 \mathrm{mg}(69 \%) ; \mathrm{Mp}=226^{\circ} \mathrm{C}-228^{\circ} \mathrm{C} ;{ }^{1} \mathrm{H}$ NMR $(500 \mathrm{MHz}$, DMSO- $\left.d_{6}\right): \delta=6.95\left(\mathrm{~d}, 1 \mathrm{H}, J=14.4 \mathrm{~Hz}, \mathrm{H}_{\mathrm{Ar}}\right), 7.35(\mathrm{t}$, $\left.1 \mathrm{H}, J=13.4 \mathrm{~Hz}, \mathrm{H}_{\mathrm{Ar}}\right), 7.47\left(\mathrm{t}, 1 \mathrm{H}, J=13.7 \mathrm{~Hz}, \mathrm{H}_{\mathrm{Ar}}\right)$, $7.87\left(\mathrm{~d}, 1 \mathrm{H}, J=13.2 \mathrm{~Hz}, \mathrm{H}_{\mathrm{Ar}}\right), 7.94(\mathrm{~d}, 1 \mathrm{H}, J=14.4 \mathrm{~Hz}$, $\left.\mathrm{H}_{\mathrm{Ar}}\right), 8.01\left(\mathrm{~d}, 1 \mathrm{H}, J=13.2 \mathrm{~Hz}, \mathrm{H}_{\mathrm{Ar}}\right), 9.02(\mathrm{~s}, 1 \mathrm{H}, \mathrm{CH})$, 10.61 (s, 1H, OH).ppm; ${ }^{13} \mathrm{C}$ NMR $\left(125 \mathrm{MHz}, \mathrm{DMSO}-d_{6}\right)$ : $\delta=116.2,122.2,122.3,124.8,125.9,126.5,132.7,133.9$, 151.4, 162.9, 166.4, 172.0 ppm; IR (KBr): $\bar{V}=3024$, 2603, 1607,1571, $1524 \mathrm{~cm}^{-1}$; MS (EI, $\left.70 \mathrm{eV}\right) \mathrm{C}_{16} \mathrm{H}_{10} \mathrm{~N}_{2} \mathrm{O}_{2} \mathrm{~S}$, $294\left(8 \%, \mathrm{M}^{++}\right), 293\left(4 \%,[\mathrm{M}-1]^{+}\right), 254(89), 253(100 \%)$. Anal. Calcd for $\mathrm{C}_{16} \mathrm{H}_{10} \mathrm{~N}_{2} \mathrm{O}_{2} \mathrm{~S}$ : C 65.29, H 3.42, N 9.51, Found C 65.20, H3.36, N 9.44.

4-(2-hydroxy-5-bromo-phenyl)-2H-pyrimido-[2,1-b]be nzothiazol-2-one (6g, $\mathrm{C}_{\mathbf{1 6}} \mathrm{H}_{\mathbf{9}} \mathrm{BrN}_{\mathbf{2}} \mathrm{O}_{\mathbf{2}} \mathrm{S}$ )

$268 \mathrm{mg}(72 \%) ; \mathrm{Mp}=173^{\circ} \mathrm{C}-174^{\circ} \mathrm{C} ;{ }^{1} \mathrm{H}$ NMR $(500 \mathrm{MHz}$, DMSO- $\left.d_{6}\right): \delta=6.98\left(\mathrm{~d}, 1 \mathrm{H}, J=14.7 \mathrm{~Hz}, \mathrm{H}_{\mathrm{Ar}}\right), 7.4(\mathrm{t}, 1 \mathrm{H}$, $\left.J=12 \mathrm{~Hz}, \mathrm{H}_{\mathrm{Ar}}\right), 7.5\left(\mathrm{t}, 1 \mathrm{H}, J=12 \mathrm{~Hz}, \mathrm{H}_{\mathrm{Ar}}\right), 7.61(\mathrm{~d}, 1 \mathrm{H}$, $\left.J=14.7 \mathrm{~Hz}, \mathrm{H}_{\mathrm{Ar}}\right), 7.93\left(\mathrm{~d}, 1 \mathrm{H}, J=13 \mathrm{~Hz}, \mathrm{H}_{\mathrm{Ar}}\right), 8.04$ (d, $\left.1 \mathrm{H}, J=10.5 \mathrm{~Hz}, \mathrm{H}_{\mathrm{Ar}}\right), 8.05\left(\mathrm{~s}, 1 \mathrm{H}, \mathrm{H}_{\mathrm{Ar}}\right) \mathrm{ppm} ;{ }^{13} \mathrm{C} \mathrm{NMR}$ (125 MHz, DMSO- $\left.d_{6}\right): \delta=110.9,119.4,121.7,122.4$, $122.7,125.4,126.8,131.8,134.1,137.6,151.2,159.5$, 163.6, $170.3 \mathrm{ppm}$; IR $(\mathrm{KBr}): \bar{V}=3100,1606,1565$, $1493 \mathrm{~cm}^{-1}$; MS (EI, $\left.70 \mathrm{eV}\right) \mathrm{C}_{16} \mathrm{H}_{9} \mathrm{~N}_{2} \mathrm{O}_{2} \mathrm{SBr}, 374\left(\mathrm{M}^{+}\right.$, $\left.\mathrm{C}_{16} \mathrm{H}_{9} \mathrm{~N}_{2} \mathrm{O}_{2} \mathrm{~S}^{81} \mathrm{Br}\right), 372\left(\mathrm{M}^{+}, \mathrm{C}_{16} \mathrm{H}_{9} \mathrm{~N}_{2} \mathrm{O}_{2} \mathrm{~S}^{79} \mathrm{Br}\right), 335$ (79), 334 (100), 333(79\%). Anal. Calcd for $\mathrm{C}_{16} \mathrm{H}_{9} \mathrm{~N}_{2} \mathrm{O}_{2} \mathrm{SBr}$ : C 51.63, H 2.44, N 7.52, Found C 51.55, H 2.39, N 7.46.

\section{Acknowledgements}

Saeed Balalaie thanks Iran National Science Foundations
(INSF) for the financial support.

\section{REFERENCES}

[1] G. P. Ellis, "Chemistry of Heterocyclic Compounds: Synthesis of Fused Heterocycles," John Wiley \& Sons, Ltd., New York, Vol. 47, 2008.

[2] A. Burger, "Medicinal Chemistry," 7th Edition, Wiley-Interscience, New York, 2010, p. 72.

[3] G. Trapani, A. Franco, G. Latrofa, A. Carotti, G. Genchi, M. Serra, G. Biggioand and G. Liso, "Synthesis and Benzodiazepine Receptor Binding of Some Imidazo- and Pyrimido[2,1-b]benzothiazoles," European Journal of Medicinal Chemistry, Vol. 31, No. 7-8, 1996, pp. 575-587. doi:10.1016/0223-5234(96)89553-5

[4] G. Trapani, A. Carotti, A. Franco, G. Latrofa, G. Gench and G. Liso, "Structure-Affinity Relationships of Some Alkoxycarbonyl-2H- or -4H-Pyrimido [2,1-b]benzothiazol-2- or 4-one Benzodiazepine Receptor Ligands," European Journal of Medicinal Chemistry, Vol. 28, No. 1, 1993, pp. 13-21. doi:10.1016/0223-5234(93)90074-O

[5] M. M. M. Gineinah, "6-, 7- and 8-(5-Aryl-1-phenyl2-pyrazolin-3-yl) Imidazo- and Pyrimido[2,1-b] Benzothiazoles as Novel Anticonvulsant Agents," Scientia Pharmaceutica, Vol. 69, No. 1, 2001, pp. 53-61.

[6] I. M. Batulin, "On the Mechanism of the Anticonvulsant Action of Some Derivatives of Pyrazole," Farmakologiya Toksikologiya, Vol. 31, No. 5, 1968, pp. 533-536.

[7] S. S. Parmar, B. R. Pandey, C. Dwivediand and R. D. Harbison, "Anticonvulsant Activity and Monoamine Oxidase Inhibitory Properties of 1,3,5 Trisubstitute dpyrazolines," Journal of Pharmaceutical Sciences, Vol. 63, No. 7, 1974, pp. 1152-1155. doi:10.1002/jps.2600630730

[8] J. J. Wade, C. B. Tose, C. J. Matson and V. L. Stelzer, "Synthesis and Antiallergic Activity of Some Acidic Derivatives of 4 H-Pyrimido[2,1- $b$ ]benzazol-4-ones," Journal of Medicinal Chemistry, Vol. 26, No. 4, 1983, pp. 608-611. doi:10.1021/jm00358a031

[9] R. J. Alaimo, "The Synthesis of Some 4H Pyrimido[2,1 b]benzothiazol 4 Ones," Journal of Heterocyclic Chemistry, Vol. 10, No. 5, 1973, pp. 769-772. doi:10.1002/jhet.5570100515

[10] A. Gupta and S. Rawat, "Synthesis and Cyclization of Benzothiazole: Review," Journal of Current Pharmaceutical Research, Vol. 3, No. 1, 2010, pp. 13-23.

[11] A. Bartovic, D. Ilavski, O. Simo, L. Zalibera, A. Belicová and M. Seman, "Synthesis of Nitro-Substituted 4-Oxo$4 H$-pyrimido[2,1-b]benzothiazole-3-carboxylic Acids and Their Spectral Characteristics," Collection of Czechoslovak Chemical Communications, Vol. 60, No. 4, 1995, pp. 583-593. doi:10.1135/cecc19950583

[12] M. A. El-Sherbeny, "Synthesis of Certain Pyrimido[2,1b]benzothiazole and Benzothiazolo[2,3-b]quinazoline Derivatives for in vitro Antitumor and Antiviral Activities," Arzneimittel-Forschung/Drug Research, Vol. 50, No. 9, 2000, pp. 848-853.

[13] J. P. Yevich, D. L. Temple, R. R. Covington, D. A. Owens, R. J. Seidehameland and K. W. Dungan, "Antial- 
lergics: 3-(1H-tetrazol-5-yl)-4H-pyrimido[2,1-b]ben-zothiazol-4-ones," Journal of Medicinal Chemistry, Vol. 25, No. 7, 1982, pp. 864-868. doi:10.1021/jm00349a020

[14] A. Kutyrev and T. Kappe, "Methanetricarboxylates as Key Reagents for the Simple Preparation of Heteroarylcarboxamides with Potential Biological Activity. Part 2[1]. Reaction of Methanetricarboxylates with 2-Aminopyridine, 2-Aminopyrimidine, 2-Aminothiazole and 2-Aminobenzothiazole," Journal of Heterocyclic Chemistry, Vol. 36, No. 1, 1999, pp. 237-240. doi:10.1002/jhet.5570360136

[15] C. Landreau, D. Deniaud, M. Evain and A. Reliquet, "Efficient Regioselective Synthesis of Triheterocyclic Compounds: Imidazo[2,1-b]benzothiazoles, Pyrimido[2,1-b]benzothiazolones and Pyrimido[2,1-b]benzothiazoles," Journal of the Chemical Society, Perkin Transactions 1, No. 6, 2002, pp. 741-745.

[16] P. J. Roy, K. Landry and Y. Leblanc, "Condensation of 2-Amino-5-chlorobenzoxazole with $\alpha$-Bromoketones: A Mechanistic Study," Heterocycles, Vol. 45, No. 11, 1997, pp. 2239-2246.

[17] Y. Tanabe, A. Kawai, Y. Yoshida, M. Ogure and H. Okumura, "Preparation of Fused Thiadiazolo- and Imidazobenzothiazoles from 2-Aminobenzothiazoles. Their Fungicidal Activity," Heterocycles, Vol. 45, No. 8, 1997, pp. 1579-1588. doi:10.3987/COM-97-7839

[18] G. Trapani, A. Frang, G. Latrofa and G. Genchi, "Synthesis and Benzodiazepine Receptor Binding of Some 4HPyrimido[2,1-b]benzothiazol-4-ones," European Journal of Medicinal Chemistry, Vol. 27, No. 1, 1992, pp. 39-44. doi:10.1016/0223-5234(92)90058-9

[19] H. Ogura, M. Kawano and T. Itoh, "Studies on Heterocyclic Compounds. XIII. Reaction of 2 Aminobenzazoles with Acetylenic Compounds," Chemical Pharmaceutical Bulletin, Vol. 21, No. 9, 1973, pp. 2019-2025. doi:10.1248/cpb.21.2019

[20] H. N. Al-Jallo and M. A. Muniem, "Synthesis and Nuclear Magnetic Resonance Spectra of Fused Pyrimidines," Journal of Heterocyclic Chemistry, Vol. 15, No. 8, 1978, pp. 849-853. doi:10.1002/ihet.5570150525

[21] C. K. Chan, J. C. N. Ma and T. C. W. Mak, "Synthesis and X-Ray Structure of Methyl 2-Oxopyrimido[2,1b]benzothiazole-4-carboxylate from Condensation of 2Aminobenzothiazole and Dimethyl But-2-ynedioate," Journal of the Chemical Society, Perkin Transactions 2, No. 8, 1977, pp. 1070-1074.

[22] J. J. Wade, R. F. Hegel and C. B. Toso, "Reaction of 2-Aminobenzazoles with Dimethyl 2-Aminofumarate. Synthesis and Nuclear Magnetic Resonance Spectroscopy of 4-Oxopyrimido[2,1-b]benzazoles," The Journal of Organic Chemistry, Vol. 44, No. 11, 1979, pp. 1811-1816. doi: $10.1021 / \mathrm{jo} 01325 \mathrm{a} 013$

[23] H. Wahe, J. T. Mbafor, A. E. Nkengfack, Z. T. Fomum, R. A. Cherkasov, O. Sterner and D. Doepp, "Heterocycles of Biological Importance: Part 7. Synthesis of Biologicallyactivepyrimido[2,1-b]benzothiazoles from Acetylenic Acids and2-Aminobenzothiazoles," Arkivoc, Vol. 2003, No. 15, 2003, pp. 107-114.

[24] A. Santagati, M. Santagati, F. Russo and G. Ronsisvalle,
"Condensed Heterocycles Containing the Pyrimidine Nucleus," Journal of Heterocyclic Chemistry, Vol. 25, No. 3, 1988, pp. 949-953. doi:10.1002/jhet.5570250347

[25] D. W. Dunwell and D. Evans "The Reactions of 2-Aminothiazoles and 2-Aminobenzothiazoles with Propiolic Acid and Its Esters," Journal of the Chemical Society C: Organic Chemistry, 1971, pp. 2094-2097.

[26] A. A. Pavlenko, Kh. S. Shikhaliev, Yu. A. Potapov and D. V. Krylsky, "Three-Component Reaction of 2-Aminobenzothiazole with Methylene-Active Carbonyl Compounds and Aldehydes," Chemistry of Heterocyclic Compound, Vol. 41, No. 5, 2005, pp. 689-690. doi:10.1007/s10593-005-0206-4

[27] A. Shaabani, A. Rahmati and S. Naderi, "A Novel OnePot Three-Component Reaction: Synthesis of Triheterocyclic 4H-Pyrimido[2,1-b]benzazoles Ring Systems," Bioorganic and Medicinal Chemistry Letters, Vol. 15, No. 24, 2005, pp. 5553-5557. doi:10.1016/j.bmcl.2005.08.101

[28] H. Ogura and T. Itoh, "Derivatives of Imidazo [2, 1-b] benzothiazole (Studies on Heterocyclic Compounds. VII)," Chemical \& Pharmaceutical Bulletin, Vol. 18, No. 10, 1970, pp. 1981-1986. doi:10.1248/cpb.18.1981

[29] A. Kreutzberger and M. Leger, "Centrally Dampening Drugs. 3rd Communication: 3-Aromatic-Aliphatically Substituted 4-Hydrox-ypyrimido[1,2- $\alpha$ ]benzimidazole-2-ones," Arzneimittel-Forschung/Drug Research, Vol. 33, No. 11, 1983. pp. 1517-1518.

[30] M. Bararjanian, S. Balalaie, F, Rominger, B. Movassagh and H. R. Bijanzadeh, "Six-Component Reactions for the Stereoselective Synthesis of 3-Arylidene-2-oxindoles via Sequential One-Pot Ugi/Heck Carbocyclization/Sonogashira/Nucleophilic Addition," The Journal of Organic Chemistry, Vol. 75, No. 9, 2010, pp. 2806-2812. doi:10.1021/j0902713x

[31] M. Bararjanian, S. Balalaie, B. Movassagh, H. R. Bijanzadeh, "Efficient Synthesis of 1,4-Disubstituted Polyfunctionalpiperazines via a Sequential One-Pot Ugi/Nucleophilic Addition Five-Component Reaction," Tetrahedron Letter, Vol. 51, No. 25, 2010, pp. 3277-3279. doi:10.1016/i.tetlet.2010.04.054

[32] M. J. Khoshkholgh, M. Lotfi, S. Balalaie and F. Rominger, "Efficient Synthesis Of Pyrano[2,3-c]coumarins via Intramolecular Domino Knoevenagel Hetero-Diels-Alder Reactions," Tetrahedron, Vol. 65, No. 21, 2009, pp. 42284234. doi:10.1016/j.tet.2009.03.032

[33] M. Bararjanian, S. Hosseinzadeh, S. Balalaie, H. R. Bijanzadeh, "Palladium Catalyzed Stereoselective Synthesis of 3-(Anilinoarylmethylene)-2-oxindoles as Hesperadin Analogues," Tetrahedron, Vol. 67, No. 14, 2011, pp. 2644 2650. doi:10.1016/j.tet.2011.02.005

[34] M. Bararjanian, S. Balalaie, B. Movassagh, F. Rominger and H. R. Bijanzadeh, "Novel and Efficient One-Pot Five- and Six-Component Reactions for the Stereoselective Synthesis of Highly Functionalized Enaminones and Dithiocarbamates," Molecular Diversity, Vol. 15, No. 2, 2011, pp. 583-594. doi:10.1007/s11030-010-9286-X

[35] M. Hadjebi, M. S. Hashtroudi, H R. Bijanzadeh and S. Balalaie, "Novel Four-Component Approach for the Syn- 
thesis of Polyfunctionalized 1,4-Dihydropyridines in Aqueous Media," Helvetica Chimica Acta, Vol. 94, No. 3,
2011, pp. 382-388. doi:10.1002/hlca.201000228 